Third Viennese Workshop on Optimal Control Theory and Analysis

20.-22. Mai 1987 - Wien, Austria

Auskunft: Prof. Dr. Gustav Feichtinger

Institut für Ökonometrie und $\mathrm{OR}$

Abteilung für $O R$

Technische Universität Wien

Argentinierstraße 8

A-1040 Wien/Austria

ISF 87 - The Seventh International Symposium on Forecasting 26. -29. Mai 1987 - Boston, USA

Auskunft: Professor J. Scott Armstrong

The Wharton School

University of Pennsylvania

Philadelphia, PA 19104, USA

OR in Transportation

1.-2. Juni 1987 - Copenhagen, Denmark

Auskunft: DORS

Sommervej 3

DK-3100 Hornbaek, Denmark

First Conference of the International Federation of Classification Societies

29. Juni-1. Juli 1987 - Aachen, FRG

Auskunft: Prof. Dr. H. H. Bock

IFCS 87

Institut für Statistik und

Wirtschaftsmathematik

Technische Universität Aachen

Wuellnerstraße 3

D-5100 Aachen, FRG
Fourth International Workshop on Computer-aided Scheduling in Public Transport

28.--31. Juli 1987 - Hamburg, FRG

Auskunft: Dr. J. R. Daduna

Hamburger Hochbahn AG

Steinstraße 20

D-2000 Hamburg 1, FRG

IFORS 87

10.-14. August 1987 - Buenos Aires, Argentinien

Auskunft: Organizing Committee Chairman

Alejandro Oliveros

Moreno $584,9^{\circ}$

1091 Buenos Aires, Argentina

IXth International Conference on Production Research

17.-20. August 1987 - Cincinnati, USA

Auskunft: Prof. Anil Mital, Conference Chairman

IXth International Conference

on Production Research

Department of Mechanical and Industrial Engineering University of Cincinnati

Cincinnati, Ohio 45221-0072, USA

1987 IEEE Conference on Management and Technology

Management of Evolving Systems

27. -30. Oktober 1987 - Norcross, USA

Auskunft: 1987 IEEE Conference on Management

and Technology

P.O. Box 920098

Norcross, GA 30092, USA

\title{
Zur Besprechung eingegangene Bücher
}

Die im folgenden aufgelisteten Bücher sind zur Besprechung im OPERATIONS RESEARCH-SPEKTRUM eingegangen. Diese Bücher können zur Besprechung angefordert werden bei: Prof. Dr. J. Schwarze, TU Braunschweig, Postfach 3329, D-3300 Braunschweig

Chamoni, P.: Simulation störanfälliger Systeme. Wiesbaden: Gabler 1986. XII, 182 Seiten. DM 68,-

Cinclar, E., Chung, K. L., Getoor, R. K. (Eds.): Seminar on Stochastic Processes, 1985. Boston Basel Stuttgart: Birkhäuser 1986. 324 Seiten. DM 98,-

Clemenz, G.: Credit Markets with Asymmetric Information. Berlin Heidelberg New York: Springer 1986. VIII, 212 pages. DM 42,-

Coelho, J. D., Tavares, L. V. (Eds.): OR Models on Microcomputers. Amsterdam: North-Holland 1986. XII, 270 pages. hfl. 180,-

Fandel, G., Grauer, M. u. a. (Eds.): Large-Scale Modelling and Interactive Decision Analysis. Berlin Heidelberg New York: Springer 1986. VIII, 363 pages. DM 64,-

Grabow, B.: Betriebliche Instandhaltung und Simulation. Frank furt: Hain 1986. 356 Seiten. DM 64,-

Hildreth, C.: The Cowles Commission in Chicago, 1939-1955. Berlin Heidelberg New York: Springer 1986. IV, 176 pages. DM 35,-

Lev, B. (Ed.): Production Management: Methods and Studies. Amsterdam: North-Holland 1986. XVI, 250 pages. hfl. 165,--
Recht, P.: Hacijan-Shor Methods and Quadratic Optimization. Frankfurt: Hain 1986. 112 Seiten. DM 32,-

Schimmelpfennig, J.: Stochastische Interaktion von Präferenzen und aggregierte Nachfrage. Frankfurt: Hain 1986. 96 Seiten. DM 29,80

Swersey, A. J., Ignall, E. J. (Eds.): Delivery of Urban Services. Amsterdam: North-Holland 1986. 408 pages. hfl. 160,-

Szidarovszky, F., Gershon, M. E., Duckstein, L.: Techniques for Multiobjective Decision Making in Systems Management. Amsterdam: Elsevier 1986. XIV, 506 pages. hfl 250,00

Tijms, H. C.: Stochastic Modelling and Analysis. A Computational Approach. Chichester New York: John Wiley \& Sons 418 pages. $£ 19.95$

Werder, Axel von: Organisationsstruktur und Rechtsnorm. Wiesbaden: Gabler 1986. 564 Seiten. DM 108,-

Wodopia, F.J.: Intertemporale Produktionsentscheidungen: Theorie und Anwendung auf die Elektrizitätswirtschaft. Frankfurt: Hain 1986. 284 Seiten. DM 58,-

Woitschach, M.: Gödel, Götzen und Computer. Stuttgart: Poller 1986. 295 Seiten. DM 39,- 